\title{
In-situ Interprofessional Perinatal Drills: The Impact of a Structured Debrief on Maximizing Training While Sensing Patient Safety Threats
}

Joy A. Greer ${ }^{1}$, Gayle Haischer-Rollo ${ }^{2}$, Donald Delorey ${ }^{3}$, Rebecca Kiser ${ }^{1}$, Timothy Sayles ${ }^{1}$, Jennifer Bailey ${ }^{1}$, Colleen Blosser ${ }^{1}$, Reginald Middlebrooks ${ }^{4}$, Christopher S. Ennen ${ }^{1}$

1. Obstetrics and Gynecology, Naval Medical Center, Portsmouth, USA 2. Pediatrics, Brooke Army Medical Center, San Antonio, USA 3. Psychology, Naval Medical Center, Portsmouth, USA 4. Anesthesiology, Naval Medical Center, Portsmouth, USA

Corresponding author: Gayle Haischer-Rollo, ghaischer@gmail.com

\section{Abstract \\ Introduction}

In-situ interprofessional emergency team training improves participants' with confidence and knowledge and identifies latent safety threats. This study examined the impact of a structured debrief on an interprofessional perinatal team's ability to identify latent safety threats and assess competency in managing perinatal emergencies. It was hypothesized that latent safety threats would be reduced and checklist compliance would increase during subsequent in-situ perinatal team training.

\section{Methods}

Two in-situ training sessions were held six months apart. The perinatal emergency response team provided care for a standardized patient with preterm twin gestation. Each session included off-ward delivery and resuscitation of the first infant, transportation to appropriate inpatient units, cesarean delivery, and resuscitation of the second twin. Postpartum hemorrhage ensued, requiring massive transfusion protocol activation. Medical experts assessed team performance with critical action checklists. A structured debrief identified latent safety threats, developed action plans, and reviewed checklist compliance. Checklist compliance rates were analyzed using a z-ratio test.

\section{Results}

The first training session: seven teams (75 staff) completed 75\% (292/391) critical action checklist items and identified 34 latent safety threats. Second training session: four teams (45 staff) completed 89\% (94/106) critical action checklist items. Ten latent safety threats were mitigated during the second session. Utilizing a z-ratio, a significant difference was detected between the overall checklist compliance rates of the two sessions, $\mathrm{z}=-3.069, \mathrm{p}=.002$. Post-hoc power calculation was $<10 \%$.

Received 10/30/2018

Review began 01/25/2019 Review ended 02/11/2019 Published 02/19/2019

(c) Copyright 2019

Greer et al. This is an open access article distributed under the terms of the Creative Commons Attribution License CC-BY 3.0., which permits unrestricted use, distribution, and reproduction in any medium, provided the original author and source are credited.

\section{Conclusions}

In-situ interprofessional perinatal emergency team training is feasible, identifies latent patient safety threats, and may improve team competency.

Categories: Medical Simulation

Keywords: simulation, multi-disciplinary

\section{Introduction}

Perinatal care accounts for $49 \%$ of inpatient care provided to female Military Health System (MHS) beneficiaries. In 2015, approximately one-third of the 43,000 deliveries in the MHS occurred at US Navy Military Treatment Facilities (MTFs) [1]. While obstetric emergencies are frequently unpredictable, improving team effectiveness significantly impacts clinical outcomes for both the mother and the infant [2]. Obstetric hemorrhage complicates $4 \%-6 \%$ of all deliveries and remains a leading cause of treatable maternal morbidity and mortality in the United States. It is also responsible for an average of one maternal death every four minutes worldwide [3].

Postpartum hemorrhage (PPH) is treatable and PPH safety programs and bundles have been associated with improved outcomes [4-5]. An integral component of these bundles includes healthcare simulation with enhanced patient scenarios and advanced task trainers targeted at improving perinatal team performance and decreasing PPH rates and, subsequently, maternal morbidity [6-8]. Institutional perinatal leadership raised concerns that latent safety threats may exist for an obstetric patient who delivers precipitously and then experiences postpartum hemorrhage prior to being admitted to the hospital and desired to test the current system navigation and processes [9-10]. 
Several studies report using in-situ simulation scenarios to identify latent safety threats within a unit or health system. These threats can be identified through the debriefing process [11-15]. Latent safety threats are "system-based threats to patient safety that can materialize at any time and are previously unrecognized by healthcare providers, unit directors, or hospital administration" [16]. Structured debriefing provides participants a unique learning opportunity to process the simulation experience, analyze thoughts, find meaning in the simulation, and better understand the connections between knowledge gained in simulation and real-life situations [17-19]. The lessons learned through the structured debriefing process can then be applied to inform patient care and hopefully improve patient outcomes. Currently, only limited studies report the use of structured debriefing during multi-disciplinary team training as a means to increase emergency checklist [20-21].

To address these concerns, the authors developed an in-situ interprofessional perinatal team simulation training scenario incorporating postpartum hemorrhage to assess system processes and team performance during a simulated perinatal emergency across the continuum of care. We hypothesized that in-situ interprofessional perinatal team simulation training would improve team function and performance. We further hypothesized that integrated formal structured debriefing would reveal unrecognized latent safety threats, identify gaps in our patient safety systems that could be addressed prior to impacting an actual patient, and increase emergency checklist compliance.

\section{Materials And Methods}

The study was determined to be Non-Human Subjects Research by the local institutional review board and was judged as exempt. The study also adhered to the framework described by the Standards for Quality Improvement Reporting Excellence (SQUIRE) guidelines [22].

\section{Study design}

The study utilized two, distinct, one-hour, in-situ interprofessional perinatal emergency simulation training scenarios, which were administered approximately six months apart in 2016. Members from the departments of Healthcare Simulation and Bioskills Training Center, Women's Health, Anesthesia, Pediatrics, and Maternal Infant Nursing at Naval Medical Center Portsmouth developed and implemented two in-situ interprofessional perinatal emergency simulation scenarios to identify potential system latent safety threats in patients with obstetric hemorrhage. The objectives were to test team communication across disciplines, determine competency in responding to perinatal emergencies, and examine emergency checklist compliance between the two training sessions. The intervention of a structured debrief was administered following the completion of the first in situ perinatal emergency simulation training scenario.

\section{Scenario for the two training sessions}

The developed scenario required the perinatal emergency response team (consisting of providers and trainees from the departments of anesthesia, obstetrics, pediatrics, nursing, and ancillary services) to provide patient care for a simulated pregnant woman with a preterm twin pregnancy who delivered the first infant off the labor and delivery unit prior to hospital admission. The team needed to transport the mother with the undelivered second twin and the newborn infant to appropriate inpatient units and deliver the second twin by cesarean delivery. The mother then experienced a postpartum hemorrhage requiring the activation of the massive transfusion protocol for the mother and emergency release of blood for the second infant. If blood transfusion was not initiated within 15 minutes after requesting the massive transfusion from the blood bank, the mother progressed to cardiac arrest and recovered with appropriate Advanced Cardiac Life Support interventions, including blood product administration. Team competency was assessed using critical action checklists by trained proctors from obstetrics, anesthesia, pediatrics, and nursing. A separate training team for each session was identified from those assigned to provide clinical care on the units for the day to prevent the in-situ training from impacting ongoing clinical care. One of the three available labor and delivery operating rooms was blocked for the operative portion of the in-situ training.

The scenario started with a standardized patient using the MamaNatalie ${ }^{\circledR}$ birthing simulator (Laerdal, Wappingers Falls, New York, United States of America) in a remote location of the hospital. A perinatal emergency drill was announced overhead. Assigned team members of the perinatal emergency response team responded to care for the patient, a 24-year-old primigravida with a twin gestation at 28 weeks gestation who was feeling the urge to push. Upon the team's arrival, the patient had preterm premature rupture of membranes followed by the delivery of the first twin. The pediatrics team used a newborn mannequin (SimNewB ${ }^{\circledR}$, Laerdal, Wappingers Falls, New York, United States of America) for the initial resuscitation of the first twin. The standardized patient was then transferred to the labor and delivery unit, as the cervix remained completely dilated with a bulging bag but no palpable, presenting fetal part. Upon the standardized patient's arrival on the labor and delivery unit and the performance of an ultrasound to discover fetal presentation (transverse, back-down), the scenario continued using a birthing mannequin (NOELLE ${ }^{\circledR}$ Maternal and Neonatal Birthing Simulator, Gaumard ${ }^{\circledR}$ Scientific, Miami, Florida, United States) modified with an insert to facilitate a cesarean delivery of the second twin and a second newborn mannequin (SimNewB ${ }^{\circledR}$, Laerdal, Wappingers Falls, New York, United States of America) to enable the resuscitation. Obstetric and anesthesia care for the maternal patient continued until the blood products arrived and began to be transfused. 
The scenario took approximately one hour to run and was followed by one hour of debrief (30 minutes within each specialty team separately: obstetrics, pediatrics, nursing, and anesthesia, as well as 30 minutes for a large group debrief to discuss team function according to TeamSTEPPS ${ }^{\mathrm{TM}}$ (Strategies and Tools to Enhance Performance and Patient Safety) principles [23]. In addition, training and system gaps that needed to be addressed were identified. Surveys were distributed to learners to assess the simulation exercise. Patient satisfaction surveys were compared six months prior to the training and five months after the identification of threats to evaluate patient perceptions of their care.

\section{Statistical analysis}

Descriptive statistics were used to determine checklist compliance rates, participant and patient satisfaction survey outcomes, latent safety threats, and participant confidence and patient satisfaction. A z-ratio test was used to determine if there was a significant change in the overall checklist compliance rates between the two training sessions. Mann-Whitney tests were used to analyze participant survey responses and an independent t-test was used to assess patient satisfaction scores. Finally, a post hoc power analysis was performed to determine the power of the study, the likelihood of the presence of a type I or type II error, and what sample size would be needed for future studies.

\section{Results}

A total of 75 staff members (seven teams) participated in the initial training over seven sessions, including representation from obstetrics, pediatrics, nursing, anesthesia, and respiratory therapy, based on hospital instruction for staffing a perinatal emergency response team. One of the eight planned training sessions was canceled due to emergent patient care needs requiring the use of the clinical spaces that had been previously assigned for in-situ simulation training.

During the first training session, proctors completed 25 checklists and 292 out of 391 (75\%) critical action checklist items were completed by the teams. The Appendix presents the checklists used for the study. Noted gaps in the critical action checklist for the obstetrics care component included quickly identifying that the standardized patient had a twin pregnancy, communication between obstetrics and pediatrics team members with the transport of standardized patient to the inpatient unit, and consideration of packing the abdomen before proceeding with hysterectomy to allow the anesthesia team to improve the simulated patient's fluid and blood product resuscitation. Role delineation and task saturation were frequent concerns for nursing. Thermoregulation and the appropriate placement of the neonate for resuscitation and the activation of emergency release blood were common gaps identified from the newborn resuscitation scenarios.

A lack of non-technical skills of the anesthesia participants was highlighted during the simulated scenarios. Anesthesia participants consisted mostly of anesthesia trainees, both anesthesiology residents and student registered nurse anesthetists. Some trainees participated by themselves and others participated with a credentialed staff member but were required to play the role of the lead provider. Once the anesthesia trainee understood the clinical situation and the necessity of the simulated parturient to go to the operating room for an emergent cesarean section, they were able to focus their actions to prepare the patient for surgery. Once inside the operating room, the trainees were given three minutes to place a spinal anesthetic in a partial task trainer. If they were unable to do so in the allotted time, the proctor stated that the fetus was decompensating and a general anesthetic must be administered. The anesthesia trainee then had to perform a rapid sequence induction and place the endotracheal tube. The most commonly missed critical steps were categorized as the non-technical skills of the anesthesia trainee. Many participants worked independently without much communication with the nursing or obstetric team. Oftentimes, when the trainee recognized he or she needed more information or help, they lacked the assertiveness to get the attention they needed. It was quickly realized by many of the anesthesia participants that other team members did not know how to provide the help or resources they needed. Help with placing additional intravenous lines, initiating the infusion of blood products, or requests to get equipment such as the rapid fluid infuser or the "hotline" was met with staff that did not know where to find the equipment or didn't know the combination to get access to secured spaces where the equipment was kept.

Through the structured debrief, the evaluation of unmet critical action items, and proctor observation, participants and proctors identified 34 latent safety threats, which were categorized as training needs, process needs, equipment needs, and visual needs. The specifics of these "needs" are presented in Tables $1-2$. The four categories of needs continued to be addressed within the hospital system and perinatal care units during this first training session. The 34 latent safety threats were prioritized and plans of action were developed to mitigate these threats. The staff was educated and corrective actions implemented. An example of the corrective action involved relocating the Belmont ${ }^{\circledR}$ Rapid Infuser (Belmont Medical Technologies, MA, US), which was moved from a locked anesthesia storage room to the primary labor and delivery operating room. This immediately improved access and decreased the delay of care. 


\section{Cureus}

\section{Training Needs}

Portable perinatal emergency bag contents

Handheld radio operations

Belmont and Ranger operations

Local anesthesia protocol

Massive transfusion protocol vs emergency release blood protocol

Use of closest elevator for transport

Communication issues/TeamSTEPPS tools

Use of Doppler to reassess fetal heart tones in transit

Blood release process $(2$ requests $=2$ runners $)$

Speed dial for operating room overhead paging

Glidescope location

Orientation to operating rooms
Process Needs

Inventory/stocking/locking of perinatal emergency bag

Security: Crowd control (Prevent inadvertent infant security code and secure elevators for transport)

Massive transfusion protocol process for unadmitted patient/computer downtime

Unadmitted patient process

Emergency Department response to perinatal emergency

Identification/color coding of team members

Baby bands added to perinatal emergency bag inventory

Ensure third Labor and Delivery operating room routinely stocked for emergencies

\section{TABLE 1: Specific Latent Safety Threats Identified Through In-Situ Perinatal Safety Simulation}

TeamSTEPPS: Team Strategies and Tools to Enhance Performance and Patient Safety

Equipment Needs

Operating room wall clocks

Markers for operating room boards

2 way radios with dedicated labor and delivery

channel/chargers

Caps/masks at operating room entrance

Laryngoscope blades/handles

Backboard

Transport gurney

Oxygen tank

Consider portable monitor

\section{Visual Needs}

Post operating room phone numbers at front desk

Blood bank/Neonatal Intensive Care Unit/front desk numbers posted in operating rooms

"Poor man's coagulation" reference in operating rooms

Local anesthesia protocol

TABLE 2: Specific Latent Safety Threats Identified Through In-Situ Perinatal Safety Simulation (cont.)

The second training session involved four teams (45 staff members). Only 15 of these staff members had participated in the initial training session. Proctors completed six checklists and 94 out of 106 (89\%) critical action checklist items were completed, for an overall checklist compliance of $89 \%$ for the second training session. Ten of the previously identified latent safety threats from the first training session were mitigated during this second training session (Table 3). Utilizing a z-ratio, a significant difference was detected between the overall checklist compliance rates between the two training sessions, $\mathrm{z}=-3.069, \mathrm{p}=.002$. However, a post hoc power analysis indicated the study lacked power $(<10 \%)$, as 84 checklists would have been needed in each group to detect a $14 \%$ improvement with $80 \%$ power. Of the 105 participants, $88(84 \%$ response rate) completed post-training surveys and self-reported that the simulation training emphasized 
the importance of communication and teamwork. The primary learning points from the in-situ simulation included reinforcement of TeamSTEPPs' principles involving team communication. On a Likert scale with anchors ( 1 =strongly disagree to $5=$ strongly agree), participants agreed or strongly agreed with the statement that the simulation training provided a team-building experience (means 4.6 and 4.44 , training sessions one and two, respectively) and would improve patient safety (means 4.67 and 4.33, training sessions 1 and 2 , respectively). Results are presented in Table 3. There was no significant difference in the mean scores between the training sessions but a majority of the participants participated in only one of the two training sessions.

\begin{tabular}{|l|l|l|}
\hline Measure & Initial Training & Subsequent Training \\
\hline Staff Participants (Teams) & $75(7)$ & $45^{\star}(4)$ \\
\hline Checklists (\% Checklist Compliance†) & $25(75 \%)$ & $6(89 \%)$ \\
\hline Latent Safety Threats Identified & 34 & 24 \\
\hline Participant Experience: Team Building, mean (median) & $4.60(5)$ & $4.44(4)$ \\
\hline Participant Experience: Improve patient safety, mean (median) & $4.67(5)$ & $4.33(4)$ \\
\hline
\end{tabular}

\section{TABLE 3: Impact of In-Situ Perinatal Simulation Training}

*Only 15 learners participated in both training events

†z-ratio

fLikert scale anchors (1=strongly disagree, 5=strongly agree); Mann-Whitney Test

Patient satisfaction surveys completed six months before the initial training were compared to the surveys completed six months after the initial training to evaluate patient perceptions of their care. Patient perceptions of their care improved by more than $5 \%$ for all assessed categories. Results are presented in Table 4. The question most germane to this study addressed the "competency of clinical staff in performing their jobs" or simply staff competency. Patient perceptions of staff competency improved from 4.6 to 4.86 , an increase of $5.65 \%$. To determine significance, a two-sample (unpaired) t-test was used. However, due to constraints with the survey data, a liberal standard deviation needed to be estimated ( $\mathrm{SD}=1.1)$ for the calculation. Results from the $\mathrm{t}$-test were significant $(\mathrm{p}=0.048)$.

\begin{tabular}{|c|c|c|c|}
\hline Measure & Six Months Before Training & Six Months After Training & $\%$ Change \\
\hline Employee/Staff Attitude, Mean Ratingł (\#responses) & $4.59(86)$ & 4.84 (424) & $+5.4 \%$ \\
\hline Caring Manner of Clinical Staff, Mean Ratingł (\#responses) & $4.55(86)$ & $4.84(424)$ & $+6.4 \%$ \\
\hline Staff competency, Mean Ratingł (\#responses) & $4.6(84)$ & $4.86(477)$ & $+5.7 \%$ \\
\hline
\end{tabular}

\section{TABLE 4: Patient Perception Ratings Before and After In-Situ Perinatal Simulation Training}

†Likert scale anchors (1=strongly disagree, $5=$ strongly agree)

\section{Discussion}

This study demonstrated the utility of structured debriefs as evident by the in-situ perinatal multidisciplinary perinatal team identifying 34 latent safety threats that could potentially impact the quality of care a patient would receive across the continuum of care. The structured debrief also allowed for the development of action plans but, most importantly, the structured debrief facilitated the mitigation of latent safety threats and improvement in emergency checklist compliance with repeated training. These findings are also consistent with previous research, which has shown that the implementation of a surgical safety checklist is associated with improvements in communication, leadership, and the ability to be assertive when necessary to improve patient safety [24].

In order to improve patient outcomes in a health system, one must look at not only the competency and performance of the team providing direct medical care but also the processes within a health system across 
the continuum of care [25]. Current techniques used to evaluate processes within a health system include leadership rounds and patient tracers [26-27]. While leadership rounds can evaluate operating procedures and the knowledge of the team prior to the provision of care, it remains difficult to evaluate the team's implementation of procedures and system processes before a patient actually interacts with the health system.

In-situ simulation training brings the simulation scenarios and drills into the clinical workspace, to improve the fidelity of the environment, system, and processes in which the health care team functions [13]. In addition to allowing the team to develop new skills or maintain infrequently used skills, a more recently reported trend uses in-situ simulation scenarios to identify latent safety threats within a unit or a health system [11-15]. These latent safety threats function as a type of lead measure on which unit and health system leadership can initiate change proactively rather than waiting for the evaluation of patient safety events to implement changes (a type of lag measure). Acting on lead measures not only addresses patient safety threats before adverse events occur but also allows increased momentum as a healthcare system becomes a high-reliability organization [28].

Challenges do exist with performing in-situ training on a busy clinical unit; however, having a smaller, separate training team from the team assigned to clinical care on the unit for the day, maintaining good communication between the training team and the team providing clinical care on the unit, and a flexible simulation team can offset those challenges and allow the successful implementation of the training. One benefit of interprofessional team training is that multiple components of team performance can be assessed during the training. While this project was significantly underpowered, we were able to gain some valuable information. Through the use of specialty-specific and interprofessional emergency checklists to assess each team, one may increase the rigor and use the number of checklists as a potential tool on which to power future studies rather than only on the number of teams that are trained, a potential limiting step at some institutions.

In-situ simulation training allows for the proactive, systematic identification of latent safety threats that can be addressed before those threats become patient safety "near misses" or sentinel events or are identified through a root cause analysis. Furthermore, in-situ training allows for the implementation of skills gained through rehearsal using task trainers and multidisciplinary team training based in a simulation center in the actual clinical environment, increasing the fidelity of the training.

The strengths of our study include the use of standardized simulation mannequins, scenarios, and emergency checklists during the in-situ training sessions. Institutional surveys were used to evaluate participant and patient experiences to determine the impact of in-situ simulation training. Formal structured debriefs were developed and implemented to identify knowledge gaps with checklist compliance and patient safety.

Our study has certain limitations. First, the design of this study resulted in a small number of groups, which was adequate to detect checklist compliance performance but had limited statistical power (post hoc power analysis $<10 \%$ ). Thus, a true difference between checklist performances between the two sessions was most likely under-detected (type II error). Second, the authors attempted to maintain scenario consistency between the two sessions by utilizing the same scenarios in both sessions. However, it is possible that the two scenarios were not entirely equal. This could have resulted in unequal checklist performance between the two groups. Third, the use of non-validated surveys for participants and patients prevent generalizability. Fourth, participant samples within each group were small and multiple surveys had to be discarded due to missing responses. Furthermore, the lack of a significant improvement in patient satisfaction is also likely due to low power. Additional limitations of our study include the sample size with a lack of paired data to determine a true pre-/post-effect in the evaluation of the participant experience.

\section{Conclusions}

The implementation of a structured debrief during in-situ perinatal interprofessional simulation training was associated with a statistically significant increase with emergency checklist compliance. Structured debriefing allowed for the identification and mitigation of latent patient safety threats during the simulation training. Results also indicate that in-situ perinatal safety training contributes to improved patient perception of staff clinical competency and staff rating of patient safety. Other medical centers may desire to develop interprofessional in-situ simulation scenarios to identify latent threats specific to their units and environment of care. They could then test process improvements within a health system as they move toward becoming high-reliability organizations with an emphasis on healthcare improvement. Further research should evaluate how to effectively implement structured debriefing into other training scenarios to evaluate improvements in teamwork and communication among training teams that stems from emergency checklist compliance, which could ultimately result in reductions in postoperative morbidity and mortality.

\section{Appendices}




\section{Cureus}

Obstetrics Critical Action Checklist (I/II)

Code Purple team arrives \& asks patient gestational age, pregnancy complications for imminent delivery

Delivers preterm infant

Hand-off ( Situation, Background, Assessment, Recommendation) to peds team

Checks to see if other twin delivery is imminent/presentation

Transfers to Labor and Delivery/Operating room

Places Twin B on monitor

Ultrasound to document fetal presentation

Communicates need for cesarean delivery but reassuring status for spinal attempt

3 minute terminal fetal bradycardia; Obstetric team communicates need to proceed with cesarean emergently under general anesthesia if spinal not yet obtained.

Cesarean delivery via low vertical/classical uterine incision- communicates to anesthesia and peds likely abruption

Poor uterine tone noted; begins postpartum hemorrhage algorithm; requests activation of massive transfusion protocol

12) Uterotonics - Medications (double pitocin, hemabate, methergine, Tranexamic acid)

13) Nonsurgical options discussed - Bakri balloon

14) Surgical options discussed/implemented (bilateral O'Leary, B-lynch, hypogastric artery ligation, prep for cesarean hysterectomy)

15) consideration of packing abdomen to wait for anesthesia to improve resuscitation.

TABLE 5: Obstetrics Critical Action Checklist 


\section{Cureus}

Obstetrics Critical Action Checklist (II/II)

Begins chest compressions when notified by anesthesia of code? Calls code blue if not already done...becomes part of ACLS team for resuscitation.

Recognizes Ventricular Fibrillation

High-quality cardiopulmonary resuscitation

Clears before analyze and shock

Immediately resumes chest compressions aftershocks

Airway management

Appropriate cycles of drug-rhythm check/shock-chest compressions

Administers appropriate drug(s) and doses

Recognizes pulseless electrical activity

Verbalizes potential reversible causes of pulseless electrical activity

Administers appropriate drug(s) and doses

Immediately resumes chest compressions after rhythm and pulse checks

Identifies return of spontaneous circulation

Ensures blood pressure/12-lead electrocardiogram is performed, oxygenation monitored, orders labs tests

Targeted temperature management

intensive care unit bed

\section{TABLE 6: Obstetrics Critical Action Checklist (Cont)}

\section{Multi-Disciplinary Neonatal Critical Action Checklist}

Twin A: Pre-term Vaginal Delivery Outside of Labor and Delivery

Initial assessment after delivery. Infant depressed with no respiratory effort.

Address thermoregulation and placement of infant for resuscitation.

Follow Neonatal Resuscitation Protocol for initial stabilization

Identify pneumothorax and treat accordingly

Apply proper use of S.T.A.B.L.E. Program

Twin B: Pre-term C-section in the Operating Room

Initial assessment after delivery. Infant depressed with no respiratory effort.

Address thermoregulation and placement of infant for resuscitation.

Follow Neonatal Resuscitation Protocol for initial stabilization

TABLE 7: Neonatal Critical Action Checklist 


\section{Cureus}

Obstetric Emergency Simulation Nursing Evaluation

1. Assesses the patient

2. Assists Provider/patient as needed a. Delivery b. locates cord clamps, scissors, blankets/towels

3. Obtain Vital signs

4. Obtains intravenous access

5. Ensures collection of blood bank specimen and its immediate transport to the blood bank

6. Explain all procedures and plans to patient

7. Effectively communicates to labor and delivery charge nurse: a. patient info b. transport to labor and delivery operating room c. immediate needs (ultrasound machine, extra personnel, delivery table, surgical tech, etc..)

8. Pre-surgical needs: a. fetal monitoring/ultrasound b. right hip roll c. antibiotics d. foley e. bovie pad f. sequential compression devices g. surgical prep

9. Abbreviated Time out a. patient name/date of birth b. procedure c. allergies

10. SBAR to neonatal team

11. Proper initiation of the massive transfusion protocol per Physician order

12. Assists Anesthesia with additional intravenous line/Cricoid Pressure

13. Demonstrates proper pad placement and operation of defibrillator

14. Ensures cancellation of the massive transfusion protocol as directed

\section{TABLE 8: Nursing Critical Action Checklist}




\section{Cureus}

Obstetric Emergency Simulation Multidisciplinary Evaluation

Team Performance

Met Not Met Comments

Shared Mental Model

1. All SBAR elements present

2. Did team "receive" the report?

3. Did all team members get report as they arrived?

Role Clarity

1. Clear Nursing and Physician Leader

2. Roles clearly established through self-assignment or team leader assigning roles?

3. Are roles clear to ALL team members

Situational Awareness

1. Ongoing monitoring and crosschecking (assesses condition and response to treatment)

2. Recognizes critical information in a timely fashion

3. Effective assertion to appropriate team member? ( 2 challenge rule if needed?)

Closed Loop Communication

1. Leader responds to input from the team

2. Read back of all orders

Psychomotor Skills/Equipment Competency

1. Quickly locates critical equipment

2. Competent with equipment and supplies

\section{TABLE 9: Multidisciplinary Evaluation}

\section{Obstetric Emergency Simulation Anesthesia Evaluation}

Critical Steps for General Anesthesia and BLOOD TRANSFUSION IN EMERGENT CESAREAN DELIVERY (I/II)
Objective Objective

Met Not Met
Comments

1. Communicates with surgeon to determine urgency of procedure

2. Assesses the patient to obtain pertinent medical and obstetric history i.e. allergies medications complications during pregnancy, vital signs surgical hx. Airway exam family anesthesia hx. Etc.

3. IF not previously done; checks equipment and monitors function/availability

4. Preoperative patient care to include left uterine displacement, sodium citrate, $100 \%$ FiO2, patent intravenous catheter, vital signs

5. Induction: Verify obstetric team readiness Apply cricoid pressure Administer induction agent and succinylcholine Direct /Video laryngoscopy Pass endotracheal tube/Inflate cuff Confirm presence of endtidal $\mathrm{CO} 2$ Notify surgeon to proceed Confirm bilateral breath sounds Release cricoid pressure Secure endotracheal tube

6. Before Delivery: Initiate mechanical ventilation Appropriate tidal volume/respiratory rate Maintain FiO2>0.5 Maintain inhaled agent > 1 MAC Protect eyes Place and suction orogastric tube Monitor temperature Assess neuromuscular blockade

TABLE 10: Anesthesia Critical Action Checklist 


\section{Cureus}

Obstetric Emergency Simulation Anesthesia Evaluation

Critical Steps for General Anesthesia and BLOOD TRANSFUSION IN EMERGENT CESAREAN DELIVERY

\section{Objective Objective \\ Met Not Met \\ Comments} (IIIII)

7. After Delivery: Initiate infusion of oxytocin Decrease inhaled agent to $<0.5$ MAC Administer nitrous oxide, opioids, and muscle relaxants as needed

8. Continually assesses patient's hemodynamic status and blood loss. Maintains constant communication with surgeon in regards to hemostasis

9. Uncontrollable bleeding: Establishes two large bore intravenous catheters if not placed already Volume resuscitation with warmed crystalloid and/or colloid Determine need for immediate transfusion Calculate total requirements Determined if crossmatched packed red blood cells or massive transfusion protocol necessary Request crossmatched packed red blood cells or massive transfusion protocol ( 6 units packed red blood cells, 4 units Fresh Frozen Plasma, 1 unit platelets) Obtain labs: Hemoglobin/Hematocrit/platelets, Prothrombin/Partial Thromboplastin, Fibrinogen, D Dimer, international normalized ratioAdminister blood products Assess ongoing bleeding Deactivate massive transfusion protocol if initiated when hemostasis occurs Repeat labs

TABLE 11: Anesthesia Critical Action Checklist (Cont)

\section{Additional Information \\ Disclosures}

Human subjects: Consent was obtained by all participants in this study. Naval Medical Center Portsmouth Institutional Review Board issued approval N/A. The study was declared exempt by the Naval Medical Center Portsmouth Institutional Review Board. Animal subjects: All authors have confirmed that this study did not involve animal subjects or tissue. Conflicts of interest: In compliance with the ICMJE uniform disclosure form, all authors declare the following: Payment/services info: All authors have declared that no financial support was received from any organization for the submitted work. Financial relationships: All authors have declared that they have no financial relationships at present or within the previous three years with any organizations that might have an interest in the submitted work. Other relationships: All authors have declared that there are no other relationships or activities that could appear to have influenced the submitted work.

\section{References}

1. Draycott T, Sibanda T, Owen L, Akande V, Winter C, Reading S, Whitelaw A: Does training in obstetric emergencies improve neonatal outcome?. BJOG. 2006, 113:177-182. 10.1111/j.1471-0528.2006.00800.x

2. AbouZahr C: Global burden of maternal death and disability . Br Med Bull. 2003, 67:1-11. 10.1093/bmb/ldg015

3. Einerson BD, Miller ES, Grobman WA: Does a postpartum hemorrhage safety program result in sustained changes in management and outcomes?. Am J Obstet Gynecol. 2015, 212:140-144. 10.1016/j.ajog.2014.07.004

4. Shields LE, Wiesner S, Fulton J, Pelletreau B: Comprehensive maternal hemorrhage protocols reduce the use of blood products and improve patient safety. Am J Obstet Gynecol. 2015, 212:272-280. 10.1016/j.ajog.2014.07.012

5. Deering SH, Chinn M, Hodor J, Benedetti T, Mandel LS, Goff B: Use of a postpartum hemorrhage simulator for instruction and evaluation of residents. J Grad Med Educ. 2009, 1:260-263. 10.4300/JGME-D-09-00023.1

6. Daniel LT, Simpson EK: Integrating team training strategies into obstetrical emergency simulation training . J Healthc Qual. 2009, 3(5):38-42. 10.1111/j.1945-1474.2009.00045.x

7. Freeth D, Ayida G, Berridge EJ, Mackintosh N, Norris B, Sadler C, Strachan A: Multidisciplinary obstetric simulated emergency scenarios (MOSES): promoting patient safety in obstetrics with teamwork-focused interprofessional simulations. J Contin Educ Health Prof. 2009, 29:98-104.

8. McGaghie WC, Draycott TJ, Dunn WF, Lopez CM, Stefanidis D: Evaluating the impact of simulation on translational patient outcomes. Simul Healthc. 2011, 6:S42-S7.

9. Deering S, Rowland J: Obstetric emergency simulation. Semin Perinatol. 2013, 37:179-188. 10.1053/j.semperi.2013.02.010

10. Egenberg S, Øian P, Bru LE, Sautter M, Kristoffersen G, Eggeb TM: Can inter-professional simulation training influence the frequency of blood transfusions after birth?. Acta Obstet Gynecol Scand. 2015, 94:316-323. 10.1111/aogs.12569

11. Wheeler DS, Geis G, Mack EH, LeMaster T, Patterson MD: High-reliability emergency response teams in the hospital: improving quality and safety using in situ simulation training. BMJ Qual Saf. 2013, 22:507-514. 10.1136/bmjqs-2012-000931

12. Siassakos D, Crofts JF, Winter C, Weiner CP, Draycott TJ: The active components of effective training in obstetric emergencies. BJOG. 2009, 116:1028-1032. 10.1111/j.1471-0528.2009.02178.x 
13. Guise JM, Lowe NK, Deering S, et al.: Mobile in situ obstetric emergency simulation and teamwork training to improve maternal-fetal safety in hospitals. Jt Comm J Qual Patient Saf. 2010, 36:443-450. 10.1016/S15537250(10)36066-1

14. Miller KK, Riley W, Davis S, Hansen HE: In situ simulation: a method of experiential learning to promote safety and team behavior. J Perinat Neonatal Nurs. 2008, 22:105-113. 10.1097/01.JPN.0000319096.97790.f7

15. Patterson MD, Blike GT, Nadkarni VM: In situ simulation: challenges and results. Advances in Patient Safety: New Directions and Alternative Approaches. Henriksen K, Battles JB, Keyes MA, et al. (ed): Agency for Healthcare Research and Quality (US), Rockville, MD; 2008. Vol. 3: Performance and Tools:

16. Patterson MD, Geis GL, Falcone RA, LeMaster T, Wears RL: In situ simulation: detection of safety threats and teamwork training in a high risk emergency department. BMJ Qual Saf. 2013, 22:468-477. 10.1136/bmjqs-2012-000942

17. Phrampus P, O'Donnell J: Debriefing using a structured and supported approach . The Comprehensive Textbook of Healthcare Simulation. Levine AI, DeMaria S Jr, Schwartz AD, Sim AJ (ed): Springer, New York; 2013. 1:73-84.

18. Morgan PJ, Tarshis J, LeBlanc V, et al.: Efficacy of high-fidelity simulation debriefing on the performance of practicing anaesthetists in simulated scenarios. Br J Anaesth. 2009, 103:531-537. 10.1093/bja/aep222

19. Fanning RM, Gaba DM: The role of debriefing in simulation-based learning. Simul Healthc. 2007, 2:115-125. 10.1097/SIH.0b013e3180315539

20. Kim JH, Kim YM, Park SH, Ju E, Choi S, Hong T: Focused and corrective feedback versus structured and supported debriefing in a simulation-based cardiac arrest team training: a pilot randomized controlled study. Simul Healthc. 2017, 12:157-164. 10.1097/SIH.0000000000000218

21. Levett-Jones T, Lapkin S: A systematic review of the effectiveness of simulation debriefing in health professional education. Nurse Educ Today. 2014, 34:e58-63. Accessed: Oct 15, 2014: 10.1016/j.nedt.2013.09.020

22. Ogrinc G, Davies L, Goodman D, Batalden P, Davidoff F, Stevens D: SQUIRE 2.0 (Standards for QUality Improvement Reporting Excellence): revised publication guidelines from a detailed consensus process. BMJ Qual Saf. 2016, 25:986-992. Accessed: September 14, 2016: 10.1136/bmjqs-2015-004411

23. Gittell JH, Beswick J, Goldmann D, Wallack SS: Teamwork methods for accountable care: relational coordination and TeamSTEPPS ${ }^{\circledR}$. Health Care Manage Rev. 2015, 40:116-120. 10.1097/HMR.0000000000000021

24. Molina G, Jiang W, Edmondson L, et al.: Implementation of the surgical safety checklist in South Carolina hospitals is associated with improvement in perceived perioperative safety. J Am Coll Surg. 2016, 222:725735. 10.1016/j.jamcollsurg.2015.12.052

25. Pronovost PJ, Holzmueller CG, Ennen CS, Fox HE: Overview of progress in patient safety. Am J Obstet Gynecol. 2011, 204:5-10. 10.1016/j.ajog.2010.11.001

26. Thomas EJ, Sexton JB, Neilands TB, Frankel A, Helmreich RL: The effect of executive walk rounds on nurse safety climate attitudes: a randomized trial of clinical units. BMC Health Serv Res. 2005, 5:46. 10.1186/14726963-5-46

27. Frankel A, Grillo SP, Pittman M, Thomas EJ, Thomas EJ, Horowitz L, Page M, Sexton B: Revealing and resolving patient safety defects: the Impact of leadership walk rounds on frontline caregiver assessments of patient safety. Health Services Research. 2008, 43:2050-2066. 10.1111/j.1475-6773.2008.00878.x

28. McChesney C, Covey S, Huling J: The 4 Disciplines of Execution: Achieving Your Wildly Important Goals . Free Press, New York; 2012. 